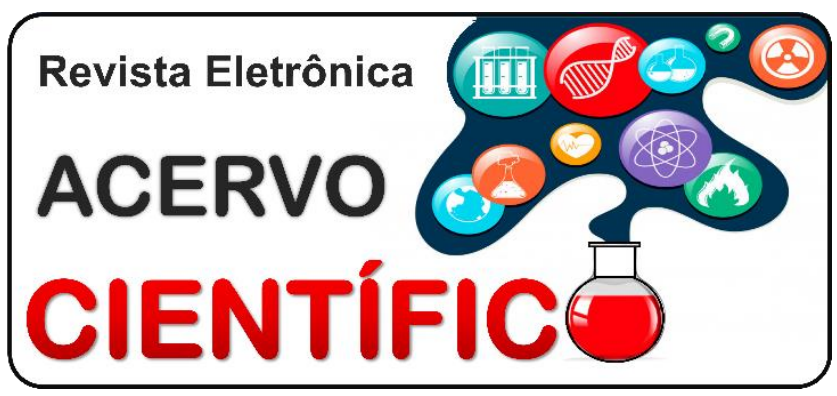

ARTIGO ORIGINAL

Recebido em: 3/2020

Aceito em: $3 / 2020$

Publicado em: 5/2020

\title{
As diversidades da predominância da sífilis congênita nas regiões do Brasil
}

Diversities of the predominance of congenital syphilis in brazilian regions

Diversidades de la predominancia de la sifilis congénita en las regiones brasileñas

Amanda Silveira Carvalho Dantas ${ }^{1 *}$, Leticia Goes Santos ${ }^{1}$, Rute de Oliveira Farias ${ }^{1}$, Thiago Lima Santos $^{1}$, Rebeca dos Santos Sirqueira ${ }^{1}$, Mayana Lula Andrade ${ }^{1}$, Roberta Souza Santos ${ }^{1}$, Izailza Matos Dantas Lopes ${ }^{1}$.

Resumo: Esse artigo buscou analisar a ocorrência de sífilis congênita nas regiões do Brasil no período de 2010-2018. A maioria dos casos da doença ocorre porque a mãe durante o pré-natal não foi testada para sífilis ou porque o tratamento preconizado pelo Ministério da Saúde não foi realizado adequadamente antes ou durante a gestação. Trata-se de um estudo que teve como instrumento de coleta de dados o Sistema de Informação de Agravos de Notificação (SINAN) do Ministério da Saúde. Foram utilizados como variáveis: zona de moradia, escolaridade, pré-natal, raça, tratamento do parceiro, classificação final da sífilis. $O$ presente estudo veio corroborar com a persistência da alta prevalência dos casos de sífilis congênita em várias regiões brasileiras. Sua ocorrência vem aumentando nos últimos anos: 2017 (24.666 casos), 2018 (26.219 casos) e estar associada ao manejo inadequado dos casos, baixo nível socioeconômico e sociodemográfico, além da baixa qualidade do pré-natal recebido pelas gestantes. Ressaltou-se também que a baixa adesão do parceiro ao tratamento esteve relacionada às causas sociais e ao aumento dos casos de sífilis congênita.

Palavras-chave: Sífilis congênita, Erradicação, Pré-natal.

\begin{abstract}
This article sought to analyze the occurrence of congenital syphilis in the regions of Brazil in the period 2010-2018. Most cases of the disease occur because the mother during prenatal care was not tested for syphilis or because the treatment recommended by the Ministry of Health was not performed properly before or during pregnancy. This is a study whose data collection instrument was the Ministry of Health's Notifiable Diseases Information System (NDIS). The variables used were area of residence, education, prenatal care, race, partner treatment, final classification of syphilis. The present study corroborates the persistence of the high prevalence of cases of congenital syphilis in several Brazilian regions. Its occurrence has been increasing in recent years: 2017 (24,666 cases), 2018 (26,219 cases) and is associated with inadequate case management, low socioeconomic and sociodemographic levels, in addition to the low quality of prenatal care received by pregnant women. It was also emphasized that the partner's low adherence to treatment was related to social causes and the increase in cases of congenital syphilis.
\end{abstract}

Keywords: Congenital syphilis, Eradication, Prenatal.

1 Universidade Tiradentes (UNIT), Aracaju - Sergipe. *E-mail: amandascd2@gmail.com

REAC/EJSC | Vol. 10 | e3373 | DOI: https://doi.org/10.25248/reac.e3373.2020 
Resumen: Este artículo buscaba analizar la aparición de sífilis congénita en las regiones de Brasil en el período 2010-2018. La mayoría de los casos de la enfermedad ocurren porque a la madre durante la atención prenatal no se le realizó una prueba de sífilis o porque el tratamiento recomendado por el Ministerio de Salud no se realizó correctamente antes o durante el embarazo. Este es un estudio cuyo instrumento de recolección de datos fue el Sistema de Información de Enfermedades Notificables (SIEN) del Ministerio de Salud. Las variables utilizadas fueron: área de residencia, educación, atención prenatal, raza, tratamiento de pareja , clasificación final de la sífilis. El presente estudio corrobora la persistencia de la alta prevalencia de casos de sífilis congénita en varias regiones brasileñas. Su ocurrencia ha aumentado en los últimos años: 2017 (24,666 casos), 2018 (26,219 casos) y se asocia con un manejo inadecuado de los casos, bajos niveles socioeconómicos y sociodemográficos, además de la baja calidad de la atención prenatal recibida por las mujeres embarazadas. También se enfatizó que la baja adherencia de la pareja al tratamiento estaba relacionada con causas sociales y el aumento de casos de sífilis congénita.

Palabras clave: Sífilis congénita, Erradicación, Cuidados prenatales.

\section{INTRODUÇÃO}

A sífilis congênita (SC) é uma doença infecciosa transmitida pela bactéria Treponema pallidum através da corrente sanguínea da gestante infectada para o concepto por via transplacentária ou, por contato direto com a lesão no momento do parto (transmissão vertical). A maioria dos casos acorre porque a mãe durante o pré-natal não foi testada para sífilis ou porque o tratamento preconizado pelo Ministério da Saúde não foi realizado antes ou durante a gestação (BRASIL, 2019).

A transmissão vertical é passível de ocorrer em qualquer fase gestacional ou estágio da doença materna e pode resultar em aborto, natimorto, prematuridade ou um amplo espectro de manifestações clínicas; apenas os casos muito graves são clinicamente aparentes ao nascimento (OMS, 2016).

É sabido que a sífilis congênita é mais frequente em grandes centros urbanos. A sua ocorrência está relacionada ao baixo nível socioeconômico, com infecção por HIV, uso de drogas, gravidez na adolescência, história de natimortalidade, comportamento sexual de risco, migração para grandes centros urbanos e, por fim, acesso limitado aos cuidados de saúde e não tratamento do parceiro infectado (MAGALHÃES DMS, et al., 2013; HILDEBRAND VLPC, 2010).

Os fatores de risco para a sífilis congênita estão associados ao acompanhamento pré-natal inadequado, onde os estudos mundiais apontam cerca de 70 a $90 \%$ dos casos encontrados. Os maiores problemas observados na assistência pré-natal são: anamnese inadequada, sorologia para sífilis não realizada nos períodos preconizados ( $1^{\circ}$ e $3^{\circ}$ trimestres), interpretação inadequada da sorologia para sífilis, falha no reconhecimento dos sinais de sífilis maternos, falta de tratamento do parceiro sexual e falha na comunicação entre a equipe obstétrica e pediátrica (TOLDO MKS et al., 2018; COSTA JS, et al., 2017).

A sífilis congênita tornou-se uma doença de notificação compulsória por meio da Portaria Ministerial no 542 de 22 de dezembro de 1986 . No período de 1998 a junho de 2019, foram notificados no SINAN 214.891 casos, refletindo o aumento dos índices de infecção da sífilis em gestante e adquirida nas mulheres em idade fértil (BRASIL, 2015).

Portanto, trata-se de uma doença que pode ser prevenida, sendo possível alcançar a eliminação da SC por meio da implementação de estratégias efetivas de diagnóstico precoce e tratamento de sífilis nas gestantes e suas parcerias sexuais. Segundo a Organização Mundial da Saúde (2016), a sífilis congênita é uma das mais graves doenças evitáveis da gestação, se realizado um pré-natal eficiente e tratamento qualificado. (OMS, 2016)

Diante do exposto objetivou-se realizar uma pesquisa para avaliar as adversidades da sífilis congênita período de 2010 a 2018 nas regiões do Brasil. 


\section{MÉTODOS}

Trata-se de estudo é transversal, observacional, retrospectivo e de caráter descritivo, tendo como instrumento de coleta de dados o Sistema de Informação de Agravos de Notificação (SINAN) do Ministério da Saúde. As informações incluídas na pesquisa são referentes aos casos notificados e confirmados de sífilis em gestante e sífilis congênita desse sistema no período de 2010 a 2018.

As variáveis utilizadas foram retiradas das fichas de investigação do SINAN da Sífilis em Gestante e da Sífilis Congênita. Dentre elas, encontram-se os dados alusivos à mãe: classificação final da sífilis, faixa etária, escolaridade, raça, zona de moradia, momento em que foi feito o diagnóstico de sífilis materna e realização ou não do pré-natal pela gestante. Ademais, tem-se os dados referentes à realização do tratamento pelo parceiro da gestante e à criança afetada pela sífilis congênita: idade no diagnóstico, classificação final da sífilis congênita. Os critérios de exclusão utilizados foram os casos de sífilis adquirida filtrados pelo próprio sistema.

O conteúdo do presente estudo é apenas documental, através de prontuário eletrônico disponível no DATASUS (Departamento de Informática do Sistema Único de Saúde do Brasil) dessa forma, são liberados o TCLE e o TALE, como também a submissão na plataforma Brasil, devido a inexistência de contato direto com os pacientes envolvidos nos dados do SINAN, com também a disponibilidade dos mesmos em uma plataforma acessível a todos.

Após a avaliação dos dados citados do SINAN eles foram inseridos em tabelas e planilhas do programa Excel. A análise estatística utilizada foi a descritiva e inferencial. As variáveis categóricas foram apresentadas por meio de frequências simples e relativas, que caracteriza a análise descritiva. Na parte inferencial, foi utilizado o teste qui-quadrado, para encontrar associação entre as variáveis. O nível de significância estatística estipulado foi de $5 \%(p \leq 0,05)$ e todos os testes foram bicaudais.

\section{RESULTADOS}

Em relação às raças, mães ditas brancas e não brancas tiveram $12,6 \%$ e $87,3 \%$ respectivamente os seus parceiros tratados. Em relação à escolaridade, mães com até 8 anos de estudo e ditas não brancas seus filhos tiveram diagnóstico de sífilis congênita em $93,9 \%$. A realização do pré-natal foi mais prevalente nas mães ditas não brancas que ocorreu em 92,4\%. Esses dados tiveram significância do $p$ quando comparados com as diversas raças (Tabela 1).

Tabela 1 - Relação entre as raças em comparação ao tratamento do parceiro, escolaridade e pré-natal das gestantes nas regiões do Brasil de 2010 a 2018.

\begin{tabular}{|c|c|c|c|c|}
\hline \multirow[b]{2}{*}{ Característica } & \multicolumn{3}{|c|}{ Raça } & \multirow[b]{2}{*}{$\mathbf{p}$} \\
\hline & $\begin{array}{c}\text { Branca } \\
\text { N (\%) }\end{array}$ & $\begin{array}{c}\text { Não branca } \\
\mathbf{N}(\%)\end{array}$ & $\begin{array}{l}\text { Total } \\
\text { N (\%) }\end{array}$ & \\
\hline Tratamento do Parceiro & & & & $<0,001$ \\
\hline Sim & $52(12,6)$ & $359(87,3)$ & $411(100)$ & \\
\hline Não & $116(69,0)$ & $2012(84,9)$ & $2128(83,8)$ & \\
\hline Escolaridade & & & & 0,002 \\
\hline Até 8 anos & $109(6,0)$ & $1680(93,9)$ & $1789(100)$ & \\
\hline 8 anos ou mais & $66(0,09)$ & $619(0,90)$ & $685(100)$ & \\
\hline Realizou pré-natal & & & & 0,008 \\
\hline Sim & $165(7,5)$ & $2032(92,4)$ & $2197(100)$ & \\
\hline Não & $20(10,8)$ & $466(18,7)$ & $486(18,1)$ & \\
\hline
\end{tabular}

Fonte: Dantas ASC, et al., 2020.

Em relação ao local de moradia, o diagnóstico de sífilis congênita predominou na zona urbana $77,4 \%$ em comparação com a zona rural $22,5 \%$. Ademais, a zona urbana apresentou $71,4 \%$ de parceiros tratados em relação a $28,5 \%$ na zona rural. O diagnóstico de sífilis nas mães de zona urbana ocorreu em $82,5 \%$ no momento do parto, e naquelas de zona rural em $17,4 \%$. Realizaram pré-natal $75,1 \%$ àquelas que residiam em zona urbana e as de zona rural $24,8 \%$ (Tabela 2). 
Tabela 2 - Relação entre a zona de moradia, a classificação da sífilis, tratamento do parceiro, faixa etária escolaridade, pré-natal e momento do diagnóstico de sífilis materna nas regiões do Brasil de 2010 a 2018.

\begin{tabular}{|c|c|c|c|c|}
\hline \multirow[b]{2}{*}{ Característica } & \multicolumn{3}{|c|}{ Zona } & \multirow[b]{2}{*}{$\mathrm{p}$} \\
\hline & $\begin{array}{c}\text { Urbana } \\
\mathbf{N}(\%)\end{array}$ & $\begin{array}{l}\text { Rural } \\
\mathrm{N}(\%)\end{array}$ & $\begin{array}{l}\text { Total } \\
\text { N (\%) } \\
\end{array}$ & \\
\hline Classificação final sífilis & & & & $<0,001$ \\
\hline Congênita & $2052(77,4)$ & $596(22,5)$ & $2648(100)$ & \\
\hline Natimorto & $165(7,0)$ & $13(2,0)$ & $178(6,0)$ & \\
\hline Descartado & $135(5,7)$ & $30(4,7)$ & $165(5,5)$ & \\
\hline Tratamento do parceiro & & & & $<0,001$ \\
\hline Sim & $318(71,4)$ & $127(28,5)$ & $445(100)$ & \\
\hline Não & $1899(85,7)$ & $458(78,3)$ & $2357(84,1)$ & \\
\hline Escolaridade & & & & $<0,001$ \\
\hline Até 8 anos & $1449(75,4)$ & $471(24,5)$ & $1920(100)$ & \\
\hline 8 anos ou mais & $626(30,2)$ & $124(20,8)$ & $750(28,1)$ & \\
\hline Realizou pré-natal & & & & $<0,001$ \\
\hline Sim & $1733(75,1)$ & $574(24,8)$ & $2307(100)$ & \\
\hline Não & $591(25,4)$ & $59(9,3)$ & $650(22,0)$ & \\
\hline Sífilis materna & & & & $<0,001$ \\
\hline Durante o pré-natal & $1050(76,4)$ & $324(23,5)$ & $1374(100)$ & \\
\hline No momento do parto & $1009(82,5)$ & $214(17,4)$ & $1223(100)$ & \\
\hline Após o parto & $287(12,2)$ & $98(15,3)$ & $385(12,8)$ & \\
\hline Não realizado & $10(0,4)$ & $50,8)$ & $15(0,5)$ & \\
\hline
\end{tabular}

Fonte: Dantas ASC, et al., 2020.

Ao se comparar a realização do pré-natal observou-se que as gestantes que realizaram, seus recémnascidos receberam o diagnóstico de sífilis congênita em $82 \%$, em detrimento daquelas que não realizaram, correspondendo a 17,9\%. O diagnóstico de sífilis materna foi realizado através das consultas de pré-natal em $60,1 \%$. Em relação aos parceiros, $94,4 \%$ foram tratados através de suas parceiras em consultas feitas durante o pré-natal (Tabela 3).

Tabela 3 - Relação entre realização do pré-natal, classificação final de sífilis congênita, sífilis materna e tratamento do parceiro nas regiões do Brasil de 2010 a 2018.

\begin{tabular}{|c|c|c|c|c|}
\hline \multirow{2}{*}{ Característica } & \multicolumn{3}{|c|}{ Realizou pré-natal } & \multirow[b]{2}{*}{$p$} \\
\hline & Sim & Não & Total & \\
\hline Classificação final sífilis & & & & $<0,001$ \\
\hline Congênita & $2177(82,0)$ & $476(17,9)$ & $2653(100)$ & \\
\hline Natimorto & $31(1,3)$ & $137(20,5)$ & $168(5,7)$ & \\
\hline Descartado & $95(4,1)$ & $55(8,2)$ & $150(5,0)$ & \\
\hline Sífilis materna & & & & $<0,001$ \\
\hline Durante o pré-natal & $1402(60,1)$ & 0 & $1402(46,9)$ & \\
\hline Durante o parto & $616(26,4)$ & $572(86,5)$ & $1188(39,7)$ & \\
\hline Após o parto & $304(13,0)$ & $85(12,9)$ & $389(13,0)$ & \\
\hline Não realizado & $9(0,4)$ & $4(0,6)$ & $13(0,4)$ & \\
\hline Tratamento do parceiro & & & & $<0,001$ \\
\hline Sim & $426(94,4)$ & $26(5,7)$ & $452(100)$ & \\
\hline Não & $1752(80,4)$ & $613(95,9)$ & $2365(84,0)$ & \\
\hline
\end{tabular}

Fonte: Dantas ASC, et al., 2020.

O SINAN notificou 214.891 casos de sífilis congênita em menores de um ano de idade, no ano de 1998 a junho de 2019 , demonstrando que $44,4 \%$ eram residentes na Região Sudeste, 30,2\% no Nordeste, $11,3 \%$ no Sul, 8,5\% no Norte e 5,6\% no Centro-Oeste. Entre os anos de 2017 a 2018, verificou-se um maior incremento nas regiões Nordeste (13,3\%), seguida das regiões Centro-Oeste $(4,3 \%)$, Sudeste $(2,9 \%)$ e Norte (1,4\%). Em 2018, observou-se uma taxa de incidência de 9,0 casos/1.000 nascidos vivos no Brasil, 
tendo as Regiões Sudeste (9,7 casos/1.000 nascidos vivos) e Nordeste (9,6 casos/1.000 nascidos vivos) mostrado as maiores taxas, ambas acima da taxa nacional. A Região Sul apresentou-se quase igual à do país (8,9 casos/1.000 nascidos vivos), enquanto as regiões Norte $(7,1$ casos/1.000 nascidos vivos) e Centro-Oeste (6,0 casos/1.000 nascidos vivos) mostraram taxas de sífilis congênita abaixo da taxa do país (Figura 1).

Figura 1 - Taxa de incidência de sífilis congênita em menores de 1 ano de idade (por 1.000 nascidos vivos) por região de residência e ano de diagnóstico. Brasil, 2008 a 2018.

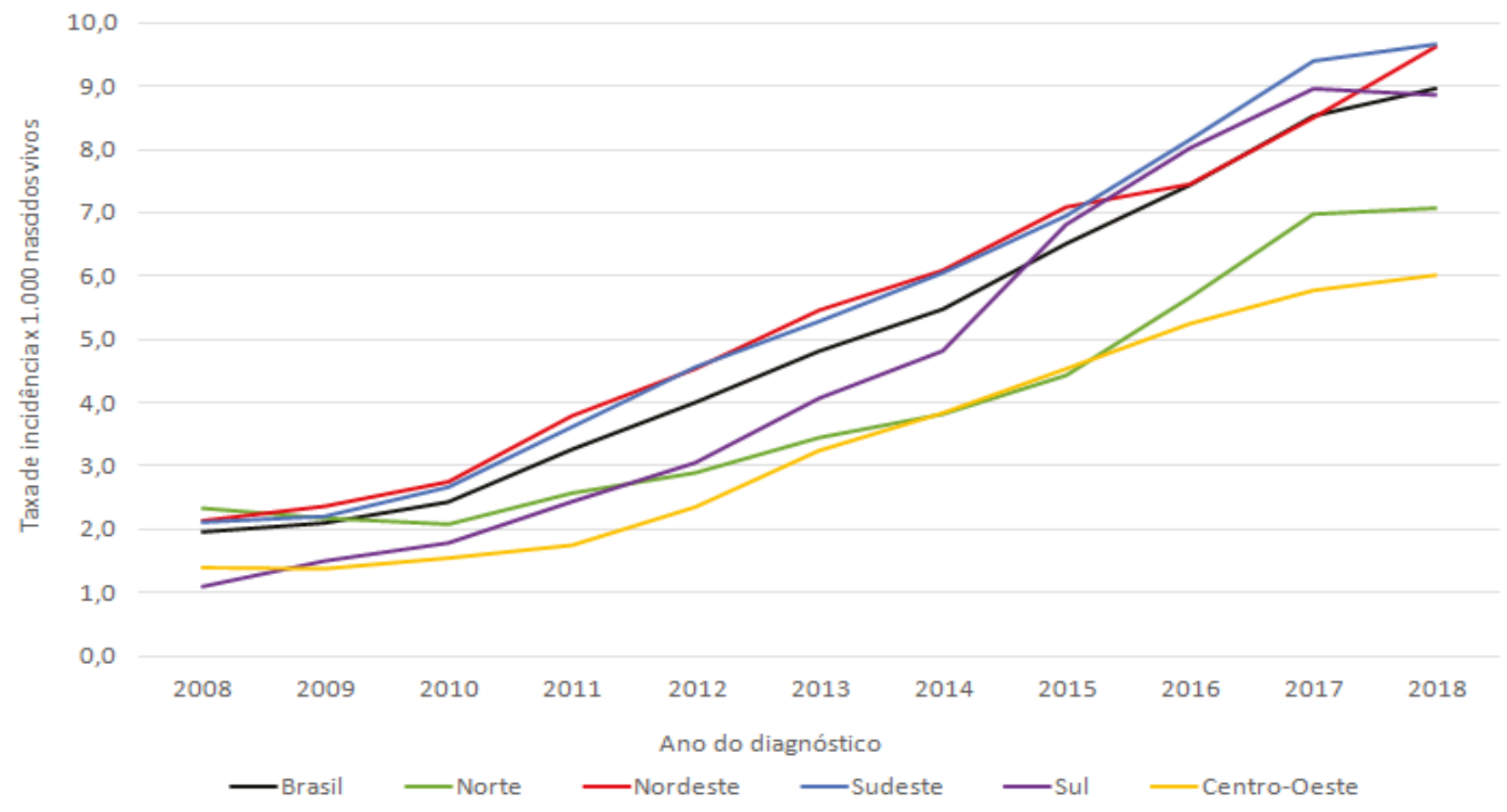

Fonte: Sistema de Informação de Agravos de Notificação (SINAN), 2019.

\section{DISCUSSÃO}

Segundo as publicações avaliadas no presente estudo constatou-se que é grande a proporção de gestantes infectadas, as quais seus filhos apresentaram sífilis congênita, possuíam baixos índices de escolaridade, de condição socioeconômica, dificuldade de acesso aos serviços de saúde, início tardio do pré-natal, além de falta de orientações sobre a doença e sobre uso de preservativos. Além desses, também foi observado o tratamento inadequado das gestantes e dos parceiros, na maioria das vezes, não recebendo ações terapêuticas recomendadas pelo Programa de Humanização do Pré-Natal, com alto índice de abandono, considerados fatores primordiais para elevar os índices da sífilis congênita (NONATO SM, et al., 2015; HILDEBRAND VLPC, 2010).

A análise do perfil clínico-epidemiológico do presente estudo verificou que mães cujos filhos possuíam sífilis congênita tinham entre 20 e 29 anos de idade $53,6 \%$, seguidas daquelas nas faixas de 15 a 19 anos $23,4 \%$ e de 30 a 39 anos 18,0\%. Estudos encontrados na literatura corroboram com os dados da pesquisa. A faixa etária predominante das gestantes com sífilis foi entre 21 e 30 anos correspondendo à idade fértil (LAFETÁ KRG, et al., 2016; LOPES IMD, et al., 2018).

Em relação às raças, mães ditas brancas e não brancas tiveram 12,6\% e 87,3\% respectivamente os seus parceiros tratados. Em relação à escolaridade, mães com até 8 anos de estudo e ditas não brancas seus filhos tiveram diagnóstico de sífilis congênita em 93,9\%. A realização do pré-natal foi mais prevalente nas mães ditas não brancas que ocorreu em 92,4\%. Esses dados tiveram significância do p quando comparados com as diversas raças. 
Em comparação ao estudo verificou-se que a relação raça/cor das mães das crianças com sífilis congênita, a maioria se declarou como parda $55,4 \%$, seguidas de brancas $25,0 \%$ e pretas $9,7 \%$ (BRASIL, 2017). Resultados semelhantes pode-se constatar em 2019 a raça/cor das mães das crianças com sífilis congênita, a maioria delas se declararam como pardas $58,4 \%$, seguidas das brancas $23,4 \%$ e pretas $8,9 \%$ (BRASIL, 2019).

A escolaridade pode-se ver que houve semelhança a estatística observada no presente estudo, pois em relação ao tempo de estudo mães que residiam em Florianópolis(SC) em 2018, (53\%) tinham mais de oito anos de estudo, (47\%) tinham menos de oito anos (TOLDO MKS, et al., 2018; COSTA JS, et al., 2017).

Dados semelhantes podem-se observar em Sergipe com mães que possuíam ensino fundamental incompleto correspondendo a $60 \%$, e $1,37 \%$ ensino superior completo e $2,7 \%$ eram analfabetas. Em Sergipe, a maioria das mães pertencia a cor parda (84,91\%) e possuíam 20-34 anos de vida (COSTA JS, et al., 2017; TOLDO MKS, et al., 2018; LOPES IMD, et al.,2018).

Ao se comparar a realização do pré-natal observou-se que as gestantes que realizaram, seus recémnascidos receberam o diagnóstico de sífilis congênita em $82 \%$, em detrimento daquelas que não realizaram, correspondendo a 17,9\%. O diagnóstico de sífilis materna foi realizado através das consultas de pré-natal em $60,1 \%$. Em relação aos parceiros, $94,4 \%$ foram tratados através de suas parceiras em consultas feitas durante o pré-natal.

A questão abordada sobre o tratamento inadequado do parceiro concomitantemente com a gestante é extremamente comum. Vários estudos epidemiológicos anteriores tem se referido a esta causa, pois concluíram que um dos motivos do não tratamento dos parceiros estavam relacionados com a falta de informação sobre a doença e sua forma de contágio, além da questão cultural do adoecer no homem, a maioria deles possuía baixa escolaridade e baixo nível socioeconômico e a maioria dos casais não utilizavam preservativos antes e após contágio (LAFETÁ KRG, et al., 2016; LOPES IMD, et al., 2018).

Em relação ao acesso ao pré-natal, em 2016, 81,0\% das mães de crianças com sífilis congênita fizeram pré-natal, enquanto $13,6 \%$ não o fizeram e $5,5 \%$ apresentaram essa informação ignorada. Em relação ao momento do diagnostico, $57,0 \%$ tiveram diagnostico de sífilis durante o pré-natal, $31,1 \%$ no momento do parto, 7,4\% apos o parto e $0,5 \%$ não teve diagnóstico (BRASIL, 2017).

Faz-se necessário melhorar a cobertura e a qualidade do pré-natal por parte da Estratégia de Saúde da Família (ESF) e de todo sistema de saúde, incluindo o parceiro sexual ativamente nas consultas de prénatal, ampliar também o diagnóstico laboratorial do Treponema pallidum e o consequente tratamento durante o pré-natal e no momento do parto. É sabido que o Ministério da saúde considera as taxas de sífilis congênita como um parâmetro de avaliação da qualidade do pré-natal e tem vinculado ao acompanhamento da gestante e à assistência ao parto, medidas que visam erradicar a sífilis congênita (COSTA JS, et al., 2017; SARACENI V, et al., 2017).

Saraceni V, et al. (2017), afirma que à situação de tratamento das gestantes que tiveram o diagnóstico no pré-natal, o tratamento foi considerado adequado em $(4,5 \%)$, inadequado em $(59,2 \%)$ e $(25,2 \%)$ não foram tratadas. Em 2018, no que concerne ao acesso ao pré-natal, $81,8 \%$ das mães de crianças com sífilis congênita fizeram pré-natal, enquanto 13,3\% não o fizeram (BRASIL, 2019).

De acordo com o Ministério da Saúde (2015), os dados mais recentes de infecção por sífilis são acometidos pelo sexo masculino, apresentando a maior taxa de portadores da doença, com $60,1 \%$ e as mulheres, em segundo lugar, com o percentual de $39.9 \%$ no período de 2010 a 2016, e a razão para esses números, é que para cada dois homens, uma mulher é infectada, e esse aumento, se dar principalmente aos seguintes fatores: a não utilização de preservativos e múltiplos parceiros (ALMEIDA L, et al., 2019; SARACENI V, et al., 2017).

Em 2017, o perfil epidemiológico do Ministério da Saúde, relatou que 62,2\% dos parceiros não foram tratados, $15,2 \%$ fizeram tratamento e para $22,6 \%$ dos casos essa informação é ignorada/em branco. 0 diagnóstico de sífilis congênita foi encontrado em $85 \%$ dos recém-nascidos cujos pais não foram tratados. 
A Organização Mundial de Saúde (OMS) estima um milhão de casos de sífilis por ano em todo o mundo entre as gestantes e preconiza a detecção e o tratamento oportunos destas e de seus parceiros sexuais portadores da sífilis, em ação conjunta com o Ministério da Saúde. Mesmo sendo de fácil diagnóstico e tratamento, ainda há dificuldades no sistema público de saúde em realizar diagnóstico precoce, principalmente em gestantes, como também tratamento adequado e em tempo hábil para que não ocorra transmissão para o feto (BRASIL, 2015).

No Brasil, em geral, nos últimos 10 anos, em especial a partir de 2010, houve um progressivo aumento na taxa de incidência de sífilis congênita: em 2006, a taxa observada era de 2,0 casos/1.000 nascidos vivos, e em 2016, a taxa observada foi maior que três vezes a taxa de 2006, passando para 6,8 casos/1.000 nascidos vivos. Em 2018, constatou-se uma taxa de incidência de 9,0 casos/1.000 nascidos vivos no Brasil, ressaltando as regiões Sudeste (9,7casos/1.000 nascidos vivos) e Nordeste (9,6 casos/1000 nascidos vivos) demonstrando serem as regiões com as maiores taxas acima da taxa nacional.

Quando observado o comportamento das taxas nos últimos 9 anos analisados (2010 e 2016), nota-se que quatro estados apresentaram queda nas taxas de detecção de sífilis congênita: Roraima, Amapá Paraíba e Alagoas. Em contrapartida os aumentos mais expressivos foram observados nos estados de Santa Catarina e Minas Gerais (BRASIL, 2017).

Em Sergipe, dados coletados nos anos de 2006 a 2018, evidenciou um crescente aumento das taxas de detecção da sífilis congênita, passando de 2,06 casos/1000 nascidos vivos, em 2006, para 8,8 casos/1000 nascidos vivos, no ano de 2018. Dados semelhantes tem-se as capitais Recife e Porto Alegre com as maiores taxas de incidência de sífilis congênita em 2018: 29,3 e 28,3 casos/1000 nascidos vivos, respectivamente, dados que representam mais de três vezes a taxa do Brasil (BRASIL, 2019).

A Portaria tornando a sífilis congênita uma doença de notificação compulsória foi em 1986, demorando doze anos para serem notificados os casos da doença no SINAN. De 1998 a junho de 2019, foram diagnosticados no SINAN 214.891 casos de sífilis congênita sendo $95.353(44,4 \%)$ residentes na região Sudeste, 64.991 (30,2\%) no Nordeste, 24.343 (11,3\%) no Sul, $18.119(8,5 \%)$ no Norte e $11.979(5,6 \%)$ no Centro-Oeste (BRASIL, 2019).

Diante do exposto, pode-se ver que a incidência de sífilis congênita está muito acima da meta preconizada pela Organização Mundial da Saúde (OMS) e Organização Pan-Americana da Saúde (OPAS) definida como ocorrência de 0,5 ou menos casos de sífilis congênita para cada mil nascidos vivos (COSTA JS, et al., 2017; TOLDO MKS, et al., 2018).

Portanto, somente por meio de um esforço unificado, com assistência pré-natal de qualidade, exames laboratoriais em tempo hábil, tratamento do casal e conscientização de todos envolvidos, pode-se alcançar o objetivo almejado de controle dessa infecção. Faz necessário utilizar da Estratégia Saúde da Família como forma de aproximação da população, conhecendo que é uma das principais portas de entrada para os serviços de saúde, e dos agentes comunitários de saúde para busca ativa às gestantes, assim investindo na educação continuada (LAFETÁ KRG, et al., 2016; LOPES IMD, et al., 2018).

\section{CONCLUSÃO}

O presente estudo veio corroborar a persistência da alta prevalência dos casos de sífilis congênita em várias regiões brasileiras, concluindo que sua ocorrência está associada ao manejo inadequado dos casos, baixo nível socioeconômico e sociodemográfico, além da baixa qualidade do pré-natal recebido pelas gestantes. Vale ressaltar também a baixa adesão do parceiro ao tratamento no que concernem várias causas sociais e o aumento dos casos de sífilis congênita naqueles que não foram tratados. Diante dessa realidade persistente no Brasil, pode-se inferir que ainda há um descaso quanto ao processo de acolhimento dessas gestantes e seus parceiros nos serviços de atenção básica. Por isso, faz de extrema importância medidas mais efetivas de prevenção e controle através dessa porta de entrada que consiste na atenção básica. 


\section{REFERÊNCIAS}

1. ALMEIDA L, et al. O pré-natal como ferramenta na prevenção da sífilis congênita: uma revisão integrativa da literatura/Pré-natal as a tool in the prevention of congenital syphilis: an integrating review of the literature. Brazilian Journal of Health Review, 2019; 2(3): 1616-1623.

2. BRASIL. Ministério da Saúde. Secretaria de Vigilância em Saúde. Departamento de DST, Aids e Hepatites Virais. Protocolo clínico e diretrizes terapêuticas para prevenção da transmissão vertical de HIV, Sífilis e Hepatites Virais. Brasília, 2015; 1ㄹed.; 272p.

3. BRASIL. Ministério da Saúde. Secretaria de Vigilância em Saúde. Guia de Vigilância Epidemiológica. Brasília, 2017; $2^{\mathrm{a}}$ ed.; 706p.

4. BRASIL. Ministério da Saúde. Secretaria de Vigilância em Saúde. Boletim Epidemiológico da Sífilis. Brasília, 2019; 44p.

5. BRASIL. Ministério da Saúde. Secretaria de Vigilância em Saúde. Protocolo Clínico e Diretrizes Terapêuticas para Atenção Integral às Pessoas com Infecções Sexualmente Transmissíveis (IST). Brasília, 2019; 122p.

6. COSTA JS, et al. Tendência temporal da sífilis congênita em Sergipe, Brasil, 2006-2017. Feira de Santana, 2019; 9: 8-15.

7. HILDEBRAND VLPC. Sífilis Congênita: fatores associados ao tratamento das gestantes e seus parceiros. Dissertação (Mestrado em Saúde Pública) - Escola Nacional de Saúde Pública Sérgio Arouca. Fundação Oswald Cruz, Rio de Janeiro, 2010; 85.

8. IZAILZA MDL, et al. Sífilis congênita em maternidade filantrópica do estado de Sergipe: ainda um desafio. 2018; $30(2): 41-46$.

9. LAFETÁ KRG, et al. Sífilis materna e congênita, subnotificação e difícil controle. Revista Brasileira de Epidemiologia, 2016; 19: 63-74.

10. MAGALHÃES DMS, et al. Maternal and congenital syphilis: a persistent challenge. Caderno de Saúde Pública, Rio de Janeiro. 2013; 29(6): 1109-1120.

11. NONATO SM et al. Sífilis na gestação e fatores associados à sífilis congênita em Belo Horizonte-MG, 2010-2013. 2015; 24: 681-694.

12. OMS. Organização Mundial de Saúde. Eliminação Mundial da Sífilis Congênita: Fundamento Lógico e Estratégia para Ação. Americas, 2016; 62p.

13. SARACENI V, et al. Vigilância epidemiológica da transmissão vertical da sífilis: dados de seis unidades federativas no Brasil. Rev Panam Salud Publica, 2017; 41: 44.

14. TOLDO MKS, et al. A recrudescência da sífilis congênita. Arquivos Catarinenses de Medicina. 2018; 47(1): 2-10. 